Trauma Berufskrankh 2008 · 10 [Suppl 3]:399-401

DOI 10.1007/s10039-008-1380-2

Online publiziert: 17. April 2008

(c) Springer Medizin Verlag 2008

\author{
A. Badke \\ Wirbelsäulenchirurgie, BG-Unfallklinik, Tübingen
}

\section{Ventrale Stabilisierung der unteren BWS/LWS}

\section{Indikation und Techniken}

Die Behandlung von Verletzungen der Wirbelsäule soll dazu führen, dass der Verletzte „seine frühere Form, Beweglichkeit und Kraft wiedererlangt". Diese Ziele wurden bereits von Lorenz Böhler [3] 1934 formuliert und sind weiterhin die Richtschnur der therapeutischen Bemühungen.

In den letzten Jahren hat sich das Spektrum der operativen Therapieoptionen in der Wirbelsäulentraumatologie erheblich erweitert. Standen zu Beginn der operativen Behandlung von Verletzungen der thorakolumbalen Wirbelsäule die dorsalen Instrumentierungen im Vordergrund, wurden in den letzten Jahren zunehmend die Indikationen zur ventralen Stabilisierung, sei es isoliert oder als dorsoventrale Fusion, herausgearbeitet [6]. Die eingeschränkte Korrelation zwischen dem radiologischen und dem klinischen Outcome führt jedoch dazu, dass die Indikationsstellung im Einzelfall im wieder problematisch sein kann $[7,8]$.

Im Folgenden soll der Stellenwert der ventralen Stabilisierung unter besonderer Berücksichtigung der minimalinvasiven Operationsverfahren näher betrachtet werden.

\section{Indikation}

Unter biomechanischen Gesichtspunkten bietet die ventrale Stabilisierung bei Berstungsfrakturen der Wirbelsäule den Vorteil, dass die Therapie direkt am „Ort der Not“, an der zerstörten ventralen Säule, ansetzt [10]. In zahlreichen Publikationen konnte belegt werden, dass die alleinige dorsale Instrumentierung von Typ-A3-, -
B- und -C-Verletzungen des thorakolumbalen Übergangs mit einem sekundären Korrekturverlust einhergeht [4, 7]. Eine besondere Rolle spielt hierbei die Zerstörung der angrenzenden Bandscheiben, die v. a. bei einer Hernierung von Nukleusgewebe in den verletzten Wirbelkörper nicht mehr in der Lage sind, nach Entfernung oder bei Versagen der dorsalen Implantate die Statik des Bewegungssegments aufrechtzuerhalten. Es ist jedoch insbesondere bei den A3.1-Verletzungen, welche durch die frühzeitige dorsale Instrumentierung optimal reponiert werden konnten, im Einzelfall schwierig, das Ausmaß des zu erwartenden Korrekturverlusts korrekt abzuschätzen. Die postoperative Magnetresonanztomographie (MRT) kann hier zusätzliche Informationen liefern.

Der zu erwartende Korrekturverlust nach dorsaler Instrumentierung stellt das Hauptargument für eine zusätzliche ventrale Stabilisierung dar. Mit der Indikation zum ventralen Vorgehen verändert sich jedoch das Therapieziel gegenüber der dorsalen Instrumentierung grundlegend. Bei der alleinigen dorsalen Instrumentierung wird nach Entfernung des Implantats die segmentale Beweglichkeit der betroffenen Segmente wieder freigegeben. Ziel der ventralen Stabilisierung ist es jedoch, eine dauerhafte Fusion der betroffenen Segmente zu erreichen [1]. In Anlehnung an die Zielvorgaben von L. Böhler ist somit festzustellen, dass durch eine ventrale Stabilisierung für die Wiederherstellung der Form Beweglichkeit geopfert wird. Insbesondere im Bereich der Lendenwirbelsäule sollte angestrebt wer- den, so wenig Bewegungssegmente wie möglich zu fusionieren, da im Langzeitverlauf nach lumbalen Fusionen in nicht unerheblicher Zahl Anschlussdegenerationen der angrenzenden Bandscheiben auftreten.

\section{Morphologische Kriterien}

Während bei den B- und C-Verletzungen die Zerstörung der betroffenen Bewegungssegmente in der Regel evident ist und eine Spondylodese rechtfertigt, stellt sich bei den dorsal instrumentierten A3.1Verletzungen immer wieder die Frage, ob nicht auch ohne eine zusätzliche ventrale Fusion eine gutes Ergebnis erreichbar ist. Bezogen auf die Klassifikation ist $\mathrm{zu}$ fordern, dass bei Frakturen, die primär der Klassifikation A1.2 oder A3.1 zugeordnet werden, eine Verletzung der dorsalen Strukturen ausgeschlossen wird. Einige Autoren empfehlen daher, bei diesen Verletzungen, wenn sie mit einem entsprechenden Kyphosewinkel einhergehen, die Durchführung einer MRT [5]. Der Stellenwert der postoperativen MRT nach dorsaler Instrumentierung zur Beurteilung der angrenzenden Bandscheibenetagen ist noch umstritten. Die postoperative Computertomographiekontrolle dokumentiert neben der korrekten Schraubenlage die Aufrichtung des Wirbelkörpers und kann weitere morphologische Daten zur Beurteilung der Heilungsprognose und Abschätzung des zu erwartenden Korrekturverlusts liefern.

Folgende morphologische Kriterien sprechen für eine ventrale Spondylodese des kranialen Bewegungssegments: 
Trauma Berufskrankh 2008 10

[Suppl 3]:399-401

DOI 10.1007/s10039-008-1380-2

c) Springer Medizin Verlag 2008

\section{A. Badke \\ Ventrale Stabilisierung der unteren BWS/LWS. Indikation und Techniken}

\section{Zusammenfassung}

Die Indikation zur additiven ventralen Spondylodese bei dorsal instrumentierten instabilen Berstungsfrakturen der thorakolumbalen Wirbelsäule ist nach wie vor umstritten. Die technische Durchführung der minimalinvasiven Verfahren ist mittlerweile weitgehend standardisiert. Bezogen auf die Indikation werden jedoch unterschiedliche Konzepte vertreten. Insbesondere aufgrund der eingeschränkten Korrelation zwischen dem radiologischen Resultat und dem klinischen Outcome konnten auch in großen Multicenterstudien keine richtungweisenden Ergebnisse erzielt werden. In der vorliegenden Arbeit sollen einzelne Aspekte der Indikationsstellung und der technischen Durchführung diskutiert werden.

\section{Schlüsselwörter}

Thorakolumbale Wirbelsäule ·Ventrale Sondylodese - Thorakoskopie - Wirbelfraktur . Versorgungsstrategie

\section{Ventral spondylodesis of the thoracolumbar spine. Indications and techniques}

\begin{abstract}
Indications for additive ventral spondylodesis of dorsal instrumented unstable burst fractures of the thoracolumbar spine are still under discussion. The technical problems of the minimally invasive procedures are mostly solved, and standardized approaches are being developed. The limited correlation between the radiologic and clinical outcomes after operative treatment of thoracolumbar fractures makes it difficult to establish standard indications even in prospective multicenter studies. This paper presents some special aspects of the indications and techniques for ventral fusion after thoracolumbar burst fractures.
\end{abstract}

\section{Keywords}

Thoracolumbar spine .

Ventral spondylodesis - Thoracoscopy .

Fractures of the spine - Treatment strategy
- persistierende Spinalkanalstenose durch dorsal verlagerte Hinterkantenfragmente

— in den Wirbelkörper herniertes Bandscheibengewebe

- Vakuumphänomen in der angrenzenden Bandscheibenetage

- ungenügende Aufrichtung des Wirbelkörpers

Eine persistierende Spinalkanalstenose nach dorsaler Instrumentierung kann und sollte im Bereich der thorakalen Wirbelsäule auch beim neurologisch unauffälligen Patienten zur Vermeidung einer sekundären Myelopathie beseitigt werden [2].

Anzeichen der Bandscheibenverletzung sowie eine ungenügende Aufrichtung des Wirbelkörpers lassen einen relevanten Korrekturverlust erwarten, sodass die Augmentation der ventralen Säule langfristig die Statik sichern und fehlstellungsbedingte Spätkomplikationen vermeiden lässt.

Wir führen nach der dorsalen Instrumentierung eine Computertomographie (CT) durch und stellen dann anhand der genannten morphologischen Kriterien unter Berücksichtigung des Alters und ggf. vorhandener Risikofaktoren die Indikation zur ventralen Spondylodese.

\section{Technik - Minithorakotomie vs. thorakoskopisches Vorgehen}

Die minimalinvasiven Verfahren zur ventralen Spondylodese sind mittlerweile als etablierte Standardverfahren zu bezeichnen, sodass sich eine detaillierte Darstellung der Operationstechnik erübrigt [2, 9]. Es haben sich jedoch 2 unterschiedliche Wege herausgebildet, deren Differenzialindikation weiterhin in der Diskussion ist.

Die minimalinvasive ventrale Spondylodese kann zum einen isoliert thorakoskopisch durchgeführt werden. Diese Technik bietet der Vorteil der kleinsten Hautinzisionen und der geringsten Traumatisierung der Thoraxwand. Außerdem kann der Situs durch die Kamera optimal eingestellt und ausgeleuchtet werden, sodass eine optimale Übersicht im Operationsgebiet erreicht wird. Nachteilig wirkt sich jedoch aus, dass während der gesam- ten intrathorakalen Operation eine 1-Lungen-Beatmung erforderlich ist. Durch eine differenzierte Beatmungstechnik kann zwar auch die nicht voll ventilierte Lunge teilweise in die Beatmung einbezogen werden, ohne dass der Operateur in seiner Sicht eingeschränkt wird, eine vollständige Beatmung ist jedoch in der Regel nicht möglich.

Bei der Minithorakotomie besteht hingegen die Möglichkeit, durch den Einsatz der vorhandenen Retraktorsysteme eine beidseitige Beatmung zu erlauben. Durch die Platzierung entsprechender Haken kann der Operationssitus so eingestellt werden, dass auch bei voller Beatmung der Lunge eine uneingeschränkte Sicht besteht. Wir führen daher bei älteren Patienten oder bei Unfallverletzten mit eingeschränkter Lungenfunktion eine Minithorakotomie durch.

\section{Ausblick}

Um eine definitive Spondylodese zu vermeiden und dennoch durch eine Augmentation der ventralen Säule eine Verringerung des Korrekturverlusts zu erreichen, wurde in letzter Zeit versucht, auch bei Berstungsbrüchen jüngerer Patienten zusätzlich zur dorsalen Instrumentierung die Kyphoplastie einzusetzen. - Abb. 1 zeigt das CT-Ergebnis bei einem uns zugewiesenen 28 Jahre alten Patienten nach einer solchen Prozedur. Neben dem unkontrollierten Zementaustritt ist festzustellen, dass keine ausreichende Wirbelkörperaugmentation erreicht und die Pathologie der angrenzenden Bandscheibe nicht behandelt wurden. Um eine dauerhafte Erhaltung des Repositionsergebnisses zu erreichen, wurde der Wirbelkörper in klassischer Weise durch einen Cage ersetzt.

$\mathrm{Ob}$ die Zementaugmentation des verletzten Wirbelkörpers durch die Entwicklung neuartiger Zemente und die Weiterentwicklung der Technik in der Behandlung von Berstungsfrakturen der thorakolumbalen Wirbelsäule zum Einsatz kommen kann, wird im Rahmen kontrollierter Studien herauszufinden sein. 


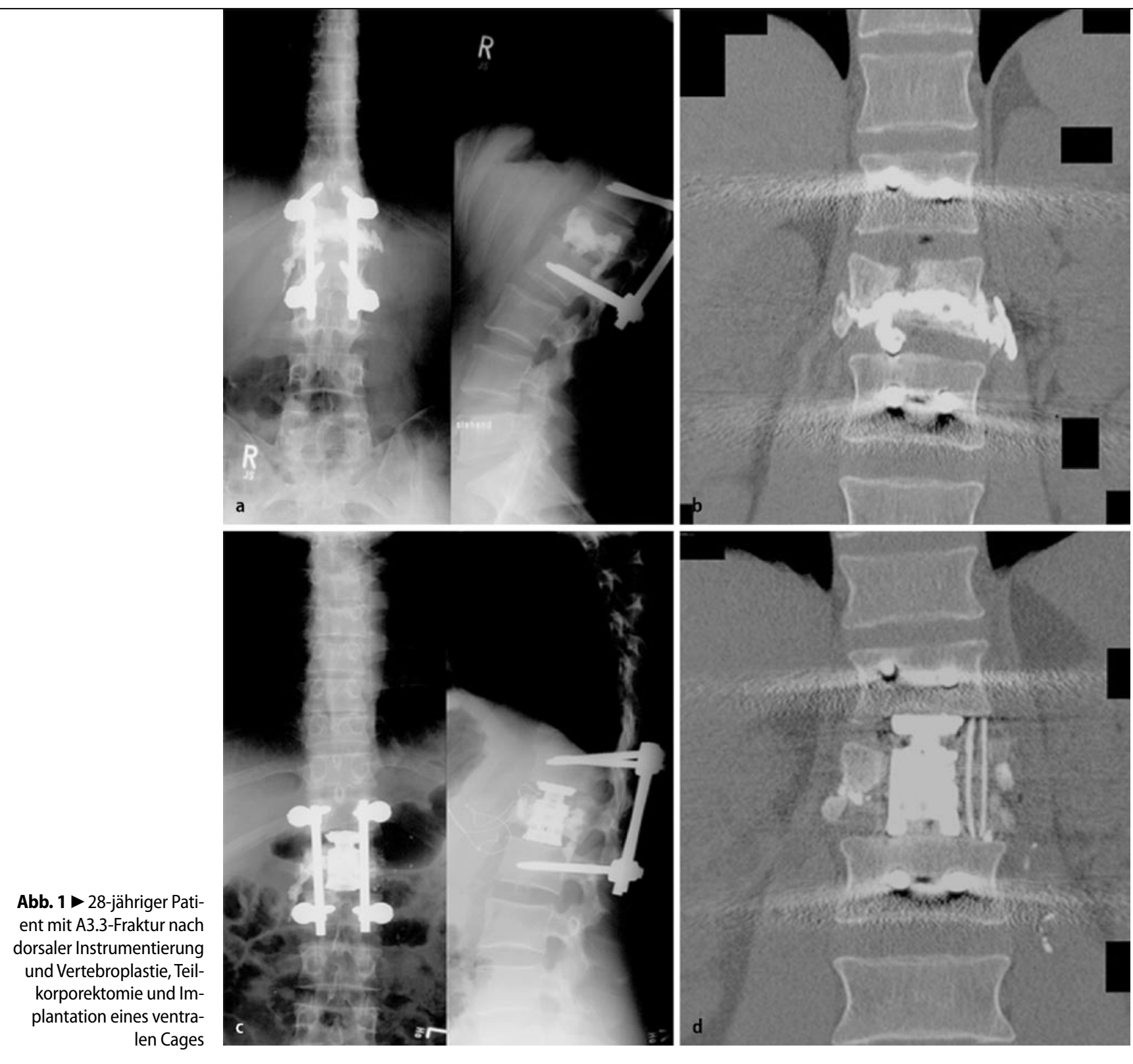

\section{Korrespondenzadresse}

\section{Dr. A. Badke}

Wirbelsäulenchirurgie, BG-Unfallklinik, Schnarrenbergstraße 95, 72076 Tübingen abadke@bgu-tuebingen.de

Interessenkonflikt. Der korrespondierende Autor gibt an, dass kein Interessenkonflikt besteht.

\section{Literatur}

1. Badke A, Jedrusik P, Feiler M et al. (2006) CT-basierter Auswertungscore nach ventraler Spondylodese bei thorakolumbalen Wirbelfrakturen. Unfallchirurg109: 119-124

2. Beisse R, Mückley R, Schmidt MH et al. (2005) Surgical technique and results of endoscopic anterior spinal canal decompression. J Neurosurg Spine 2: 128-136
3. Böhler $L$ (1938) Wirbelbrüche und Wirbelverren kungen. In: Böhler L (Hrsg) Die Technik der Knochenbruchbehandlung, Bd 1. Maudrich, Wien

4. Eysel P, Rompe JD, Hopf C et al. (1994) Die Bedeutung der Bandscheibe für den Repositionsverlust operativ stabilisierter Frakturen der Rumpfwirbelsäule. Unfallchirurg 97: 451-457

5. Haba H, Taneichi H, Kotani Y et al. (2003) Diagnostic accuracy of magnetic resonance imaging for detecting posterior ligamentous complex injury associated with thoracic and lumbar fractures. J Neurosurg 99: 20-26

6. Knop C, Blauth M, Bühren V et al. (2000) Operative Behandlung von Verletzungen des thorakolumbalen Übergangs. Teil 2: Operationen und röntgenologische Befunde. Unfallchirurg 103: 1032-1047

7. Knob C, Blauth M, Büren V et al. (2001) Operative Behandlung von Verletzungen des thoraco-lumbalen Überganges Teil 3: Nachuntersuchungen. Ergebnisse einer prospektiven multizentrischen Studie der Arbeitsgemeinschaft "Wirbelsäule" der Gesellschaft für Unfallchirurgie. Unfallchirurg 1004 583-600
8. Siebenga J, Leferink VJ, Segers MJ et al. (2006) Treatment of traumatic thoracolumbar spine fractures: a multicenter prospective randomized study of operative versus nonsurgical treatment. Spine 31: 2881-2890

9. Verheyden AP, Hoelzl A, Lill H et al. (2004) The endoscopically assisted simultaneuos postero-anterior reconstruction of the thoracolumbar spine in prone position. Spine J 4: 540-549

10. Wilke HJ, Kemmerich V, Claes LE et al. (2001) Combined antero-posterior spinal fixation provides superior stabilisation to a single anterior or posterior procedure. J Bone Joint Surg Br 83-B: 609-617 